\title{
A Trial for an Evaluation of Perianeurysmal Arterial Pressure Change during Carotid Artery Stenting in Patients with Concomitant Severe Extracranial Carotid Artery Stenosis and Ipsilateral Intracranial Aneurysm
}

\author{
Daisuke WAJIMA¹, Ichiro NAKAGAWA ${ }^{1}$, Takeshi WADA², Hiroyuki NAKASE ${ }^{1}$ \\ ${ }^{1}$ Nara Medical University, Department of Neurosurgery, Kashihara, Nara, Japan \\ ${ }^{2}$ Nara Medical University, Department of Radiology, Kashihara, Nara, Japan \\ Corresponding author: Daisuke WAJIMA wajima@naramed-u.ac.jp
}

\section{ABSTRACT}

Concomitant occurrence of severe extracranial large vessel stenosis and an ipsilateral intracranial aneurysm is often detected incidentally, but the optimal therapeutic strategy is not clear. Several reports have investigated whether simultaneous carotid artery stenting (CAS) and coil embolization, or multi-stage therapy is better. These treatment strategies are still controversial because simultaneous therapy will decrease the risk of aneurysm rupture, but may also increase the risk of thromboembolic complications. For multi-stage therapy, the interval between CAS and coil embolization for the ipsilateral intracranial aneurysm remains obscure. We experienced a case of severe extracranial internal carotid artery stenosis, ipsilateral intracranial aneurysm. Multi-stage therapy was chosen, and perianeurysmal arterial pressure did not increase over $100 \mathrm{mmHg}$ after CAS. Subsequent ipsilateral coil embolization was performed 3 weeks later with careful blood pressure control to prevent aneurysmal rupture and with the expectation of stable neointimal formation over the stent strut to prevent thrombotic complications during coil embolization. All procedures were performed without any complications. We suggest that evaluation of perianeurysmal arterial pressure may be helpful for selecting a treatment strategy in patients with multiple ipsilateral lesions to prevent thromboembolic and hemorrhagic complications.

KEYWORDS: Ipsilateral unruptured aneurysm, Perianeurysmal arterial pressure, Severe extracranial carotid artery stenosis

\section{INTRODUCTION}

$\mathrm{T}$ The concomitant occurrence of severe extracranial carotid artery stenosis and an ipsilateral intracranial aneurysm is often detected, however, the optimal therapeutic strategy is not clear $(4,6,12)$.

Here we describe a case report in which coil embolization was performed 3 weeks after carotid artery stenting (CAS) and in which we evaluated arterial pressure of the parent artery near the ipsilateral aneurysm during CAS. We discuss the safety of multi-stage therapy for simultaneous CAS for severe carotid artery stenosis and coil embolization for an ipsilateral intracranial aneurysm, and the role of perianeurysmal arterial pressure measurement for selecting a treatment strategy.

\section{CASE REPORT}

A 62-year-old female with numbness in her right hand was diagnosed with severe symptomatic left internal carotid artery (ICA) origin stenosis and an unruptured left ICAposterior communicating artery (IC-Pcom A) aneurysm (size $7.5 \times 9.0 \times 6.8 \mathrm{~mm})$. The carotid stenosis lesion was $95 \%$ stenosis (North American Symptomatic Carotid Endarterectomy Trial (NASCET) classification) at the $\mathrm{C} 2 / 3$ vertebral level.
Daisuke WAJIMA (D) : 0000-0001-8370-9864 Ichiro NAKAGAWA (D) : 0000-0002-1340-3293
Takeshi WADA (D): 0000-0002-3663-553X

Hiroyuki NAKASE (D) : 0000-0002-2726-5960 
Dual antiplatelet therapy that comprised aspirin (100 mg/day) and clopidogrel (75 mg/day) was initiated at least 4 weeks before CAS, and statins were administered.

Carotid stenosis was very severe for revascularization to prevent hemodynamic cerebral ischemia, and the right ICA aneurysm was over $5 \mathrm{~mm}$, which was too large to be cured to prevent rupture, according to the results of "The Natural Course of Unruptured Cerebral Aneurysms in a Japanese Cohort" (UCAS Japan) (13). Although several therapeutic strategies were considered for treating these lesions, the patient selected multi-stage interventional therapy after informed consent.

Initially, CAS was performed under local anesthesia. An 8Fr OPTIMO balloon guiding catheter (Tokai Medical Products Inc., Kasugai, Aichi, Japan) was introduced just proximal to the cervical stenotic lesion via the right femoral artery (Figure 1A). Distal protection was performed with Spider (ev3 Endovascular, Plymouth, MA, USA), and concomitant proximal protection was performed with an 8Fr OPTIMO balloon guiding catheter. Proximal balloon protection was performed at the lesion cross and for arterial pressure measurement. Stent selection was based on assessments of the virtual histology of the plaques with intravascular ultrasound, and the Carotid Wall stent $8 \times 29$ $\mathrm{mm}$ (Boston Scientific, Natick, MA, USA) system was used. Pre-dilation with Jackal Rx $3.0 \times 30 \mathrm{~mm}$ (Kaneka Medics, Osaka, Japan) (Figure 1B) and post-dilation with Aviator Plus $4.0 \times 30 \mathrm{~mm}$ (Cordis, Miami, FL, USA) were performed. Both before and after CAS, the mean arterial pressure at the level of the CCA (pre-CAS $113 \mathrm{mmHg}$, post-CAS $70 \mathrm{mmHg}$ ), at the petrous level (pre-CAS $36 \mathrm{mmHg}$, post-CAS $47 \mathrm{mmHg}$ ), and at the C2/C3 vertebral level (pre-CAS $42 \mathrm{mmHg}$, postCAS $44 \mathrm{mmHg}$ ) was measured with Excelsior SL-10 (Boston Scientific). After the CAS procedure, we confirmed dilation of the carotid artery using angiography (Figure 1C). She was discharged without any perioperative complications. In addition, her magnetic resonance imaging (MRI) diffusionweighted image (DWI) showed no new ischemic lesions.

Second, coil embolization for the right IC-Pcom aneurysm was performed under general anesthesia 3 weeks after CAS. Aneurysmal growth and CAS-related plaque protrusion/ thrombosis were not observed 3 weeks after CAS. A 7Fr FUBUKI $90 \mathrm{~cm}$ STR guiding catheter (ASAHI Intec Inc., Tokyo, Japan) was introduced into the right ICA distal to the CAS portion via the right femoral artery (Figure 1D). After a Sceptor C balloon catheter $4 \mathrm{~mm} \times 10 \mathrm{~mm}$ (Terumo Inc., Tokyo, Japan) was positioned in the parent artery distal to the right ICA aneurysm, an Excelsior SL-10 pre-shaped to $45^{\circ}$ was introduced into the aneurysm (Figure 1E). After the coil embolization procedure (Figure 1F), we confirmed complete occlusion of the aneurysm using angiography (Figure 1G). She was discharged without any perioperative complications, and her MRI DWI showed no new ischemic lesions.

\section{DISCUSSION}

Detecting the coexistence of severe extracranial carotid artery stenosis and asymptomatic ipsilateral intracranial aneurysm leads to a quandary regarding the management $(1,3,7)$. Some authors reported that untreated aneurysms in patients who underwent carotid revascularization may rupture or regrow after several months or years $(8,9)$. However, no evidence is available about how to select a treatment strategy in patients with these lesions.

A single-stage approach has the advantage that the procedure takes only a day, can reduce the risk of aneurysmal rupture due to increased cerebral blood flow (CBF) following CAS. The single-stage approach, however, may involve risks of ischemic events including in-stent thrombosis during coil embolization followed by CAS $(1,3)$.

Therefore, we prefer the multi-stage strategy for multiple ipsilateral lesions. However, unresolved issues remain such as which patients are not suitable for the multi-stage strategy, how to determine the strategy for prevention of aneurysmal rupture. For resolution of these issues, we propose evaluation of the arterial pressure of the parent artery near the ipsilateral aneurysm during CAS.

Recent hemodynamic analysis based on computational fluid dynamics revealed that the arterial pressure near the aneurysm neck can be over $140 \mathrm{mmHg}$ several hours before an aneurysmal rupture (11). Therefore, we speculate that the evaluation of perianeurysmal arterial pressure change during CAS would be helpful for prediction of aneurysmal rupture and may provide important information for selecting the treatment strategy. In the present case, we measured the arterial pressure and the mean perianeurysmal arterial pressure was $42 \mathrm{mmHg}$ before CAS and $44 \mathrm{mmHg}$ after CAS.

Regarding the optimal interval for coil embolization after CAS, no evidence exists regarding the optimal interval for multistage endovascular therapy. The mechanisms of in-stent thrombosis involve an inflammatory response from the vessel wall, and leukocyte accumulation leads to thromboembolic complications following balloon injury or stent implantation, especially when a bare-metal stent strut is exposed (10). Therefore, stable neointimal coverage over the stent may be required to prevent these thromboembolic complications during coil embolization following CAS (2). Stable neointimal formation over the stent strut is typically considered to be complete by 14-28 days post-stenting (5). We suggest that the optimal interval is over 3 weeks for coil embolization following CAS.

\section{- CONCLUSION}

Evaluation of perianeurysmal arterial pressure before and after CAS may be helpful for the multi-stage endovascular treatment approach for treatment of severe extracranial carotid artery stenosis and a concomitant unruptured ipsilateral intracranial aneurysm. 


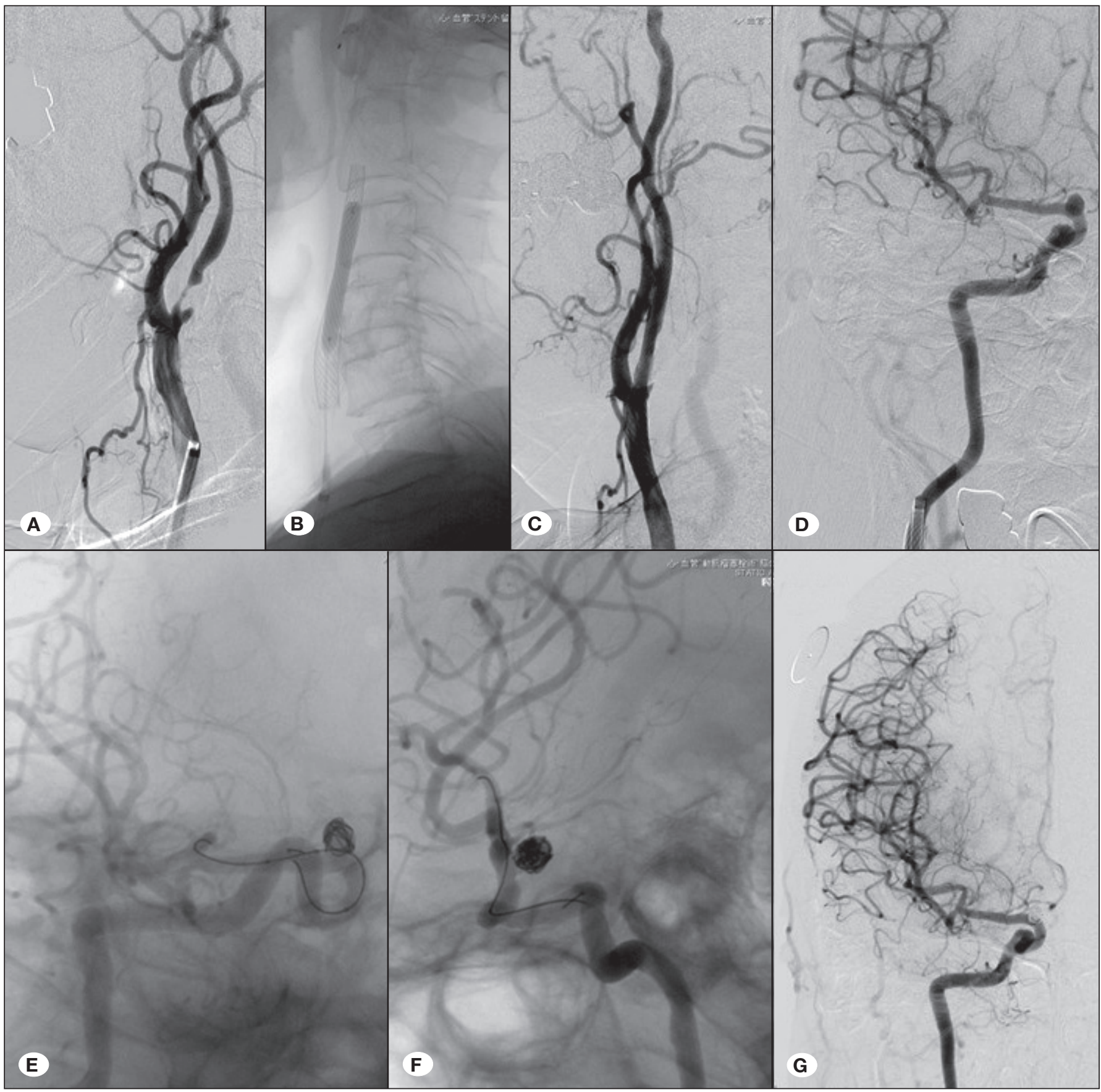

Figure 1: Presentation of the case. A balloon guiding catheter was introduced just proximal to the cervical stenotic lesion (A). Distal filter protection was performed, and concomitant proximal protection was performed with a balloon guiding catheter. Pre-dilation and post-dilation angiographies (B) were performed. After the CAS procedure, we confirmed dilation of the carotid artery using angiography (C). Next, coil embolization was performed 3 weeks after CAS. A guiding catheter was introduced into the left ICA via the stent (D). After a balloon catheter was positioned in the parent artery distal to the left ICA aneurysm (E), a micro-catheter was introduced into the aneurysm. Framing coiling was performed, and then packing was performed (F). After the coil embolization procedure, we confirmed complete occlusion of the aneurysm using angiography (G). PTA and stent replacement for left subclavian artery occlusion were performed 1 month after the coil embolization. 


\section{REFERENCES}

1. Badruddin A, Teleb MS, Abraham MG, Taqi MA, Zaidat OO: Safety and feasibility of simultaneous ipsilateral proximal carotid artery stenting and cerebral aneurysm coiling. Front Neurol 1: 120, 2010

2. Douglas G, Kampen EV, Hale AB, McNeill E, Patel J, Crabtree MJ, Ali Z, Hoerr RA, Alp NJ, Channon KM: Endothelial cell repopulation after stenting determines in-stent neointima formation: Effects of bare-metal vs. drug-eluting stents and genetic endothelial cell modification. Eur Heart J 34(43): 3378-3388, 2013

3. Gallego León JI, Concepción Aramendía L, Ballenilla Marco F, Vázquez Suárez JC: Concomitant endovascular treatment of concomitant extracranial carotid stenosis and intracranial aneurysm. Our experience. Interv Neuroradiol 15: 53-59, 2009

4. Iwata T, Mori T, Tajiri H: Successful staged endovascular treatment of a symptomatic cervical carotid bifurcation stenosis coupled with a coincidental unruptured cerebral aneurysm in the carotid distal segment. AJNR Am J Neuroradiol 29: 1948-1950, 2008

5. Nakagawa I, Wada T, Park HS, Nishimura F, Yamada S, Nakagawa $\mathrm{H}$, Kichikawa $\mathrm{K}$, Nakase $\mathrm{H}$ : Platelet inhibition by adjunctive cilostazol suppresses the frequency of cerebral ischemic lesions after carotid artery stenting in patients with carotid artery stenosis. J Vasc Surg 59(3): 761-767, 2014

6. Navaneethan SD, Kannan VS, Osowo A, Shrivastava R, Singh S: Concomitant intracranial aneurysm and carotid artery stenosis: A therapeutic dilemma. South Med J 99: 757-758, 2006
7. Park JC, Kwon BJ, Kang HS, Kim JE, Kim KM, Cho YD, Han $\mathrm{MH}$ : Single-stage extracranial carotid artery stenting and intracranial aneurysm coiling: Technical feasibility and clinical outcome. Interv Neuroradiol 19: 228-234,2013

8. Sasaki H, Wajima D, Aketa S: Case report and review of literature: Coil embolization for the ipsilateral unruptured internal carotid artery aneurysm after carotid arterial stent for the symptomatic internal carotid stenosis. Curr Pract Neurosurg (Japanese) 1: 86-90, 2017

9. Siddiqui A, Vora N, Edgell RC, Callison RC, Kitchener J, Alshekhlee A: Rupture of a cerebral aneurysm following carotid endarterectomy. J Neurointerv Surg 4: e27, 2012

10. Simon DI: Inflammation and vascular injury: Basic discovery to drug development. Circ J 76(8): 1811-1818, 2012

11. Sudhir BJ, Reddy JB, Menon G, Callison RC, Kitchener J, Alshekhlee A: Computing the difference between life and death: Prerupture blood flow analysis of a fatal aneurysm bleed. Neurol India 64 Suppl: 98-100, 2016

12. Suh BY, Yun WS, Kwun WH: Carotid artery revascularization in patients with concomitant carotid artery stenosis and asymptomatic unruptured intracranial artery aneurysm. Ann Vasc Surg 25: 651-655, 2011

13. UCAS Japan Investigators; Morita A, Kirino T, Hashi K, Aoki N, Fukuhara S, Hashimoto N, Nakayama T, Sakai M, Teramoto A, Tominari S, Yoshimoto T: The natural course of unruptured cerebral aneurysms in a Japanese cohort. N Engl J Med 366(26): 2474-2482, 2012 